\title{
Glutathione enhances fibroblast collagen contraction and protects keratinocytes from apoptosis in hyperglycaemic culture
}

\author{
M. Deveci, R.R. Gilmont, ${ }^{\star}$ W.R. Dunham, $\dagger$ B.P. Mudge, ${ }^{\star}$ D.J. Smith^ and C.L. Marcelo* \\ Department of Plastic and Reconstructive Surgery, Gulhane Military Medical Academy, Ankara 06018, Turkey \\ *Department of Plastic and Reconstructive Surgery and †Biophysics Research Division, University of Michigan, Medical Center, Kresge I R 5659, Ann Arbor, MI \\ 48109, U.S.A.
}

\section{Summary}

\section{Correspondence \\ Cynthia Marcelo. \\ E-mail: cmarcelo@umich.edu}

\section{Accepted for publication \\ 16 June 2004}

Key words:

apoptosis, diabetes, fibroblast contraction,

glutathione, wound healing

\section{Conflicts of interest:}

None declared.
Background Cutaneous wound healing is relatively slow in patients with diabetes. Objectives To test the hypothesis that this defect in healing of wounds in patients with diabetes results from dysfunction of skin fibroblasts and epidermal keratinocytes and that this dysfunction is related to disrupted intracellular glutathione (GSH) homeostasis.

Methods We investigated the effects of esterified GSH on the contraction of fibroblasts in a fibroblast-populated collagen lattice and on keratinocyte apoptosis.

Results High glucose medium (hyperglycaemia) reduced the contraction ability of fibroblasts $(P<0.05)$. The normalization of glucose medium concentrations for hyperglycaemic fibroblasts did not restore the contraction capacity. The percentage of apoptotic keratinocytes was statistically higher in hyperglycaemic cells $(\mathrm{P}<0 \cdot 05)$. GSH media concentrations ranging from $0 \cdot 1$ to $100 \mu \mathrm{mol} \mathrm{L}^{-1}$ restored the ability of hyperglycaemic fibroblasts to contract the gels in a concentration-dependent manner. Primary human keratinocytes grown in hyperglycaemic medium were more susceptible to apoptosis, and treatment with esterified GSH rescued the keratinocytes from apoptosis.

Conclusions These data suggest that intracellular GSH can normalize skin cell functions disrupted by in vitro cell growth under hyperglycaemic conditions.
Successful wound healing is accomplished by fibroplasia, angiogenesis and re-epithelialization, processes which involve the migration and proliferation of fibroblasts, endothelial cells and epithelial cells. ${ }^{1-3}$ Wound contraction is an important part of healing and occurs via mechanisms reportedly mediated by a specialized form of fibroblast, the myofibroblast, using an undefined mechanism that differs from that seen in smooth muscle contraction. ${ }^{2-4}$

The chronic nonhealing dermal ulcer is one of the most significant and challenging problems in the management of patients with diabetes. ${ }^{1}$ In this type of delayed wound healing, insufficient granulation tissue formation, insufficient epithelialization and lack of contraction probably result from defects in fibroblast function. ${ }^{5}$ Oxidative stress is proposed as an important pathogenic factor in diabetic wound complications and appears to affect cell replication and life span. In vitro antioxidant therapy is able to reverse the impaired proliferative activity of different cell types from diabetic skin. ${ }^{6}$ However, there are no reported studies on the effect of oxidative stress on fibroblast contraction.
Other investigators have suggested that high glucose levels induce programmed cell death (apoptosis) in vitro ${ }^{5,7-10}$ via oxidative stress mechanisms. Oxidative stress is suggested to increase free oxygen radicals, coupled with a decreased activity of radical scavengers, especially glutathione (GSH).

The GSH redox cycle is a primary component of the cellular protective mechanism against oxidant stress. ${ }^{11,12} \mathrm{GSH}$ is a low-molecular-weight tripeptide ( $\gamma$-glu-cys-gly) with two biologically important structural features: a thiol group and a $\gamma$-glutamyl linkage. It serves as electron acceptor for hydrogen peroxides and becomes toxic, oxidized thiol (GSSG). The rates of introduction (synthesis, uptake) and the rates of elimination (conjugation, efflux) determine the overall cell redox balance. A previous clinical study has shown that tissue samples from diabetic wounds have low levels of GSH and high levels of mixed protein disulphides and of GSSG compared with uninjured skin sites. ${ }^{13}$

In the present study, primary cultures of human dermal fibroblasts were used to test the hypothesis that the lack of wound contraction in patients with diabetes is a result of 
fibroblast dysfunction and that the defective fibroblast contraction is related to disrupted intracellular GSH homeostasis. Bell et al. ${ }^{14}$ first reported the construction and use of a fibroblast-populated collagen lattice (FPCL) as an in vitro contraction model. Considerable evidence has implicated direct cellular traction as the means by which fibroblasts and other types of cells contract collagen lattices. The contraction of collagen lattice needs a complex cell-extracellular matrix interaction involving integrins, focal adhesions and an intact cytoskeleton. ${ }^{1}$ Thus, the contraction of FPCL and the effect of GSH treatment on FPCL contraction were tested in this study.

\section{Materials and methods}

\section{Cell culture}

\section{Keratinocytes}

Primary epidermal cells (keratinocytes) were cultured from breast reduction skin of healthy women. ${ }^{15}$ After overnight trypsinization of the split-thickness skin, the resulting epidermal cell suspension was plated in MCDB 153 with $0.218 \mu \mathrm{g} \mathrm{mL}^{-1}$ hydrocortisone, $5 \mu \mathrm{g} \mathrm{mL}^{-1}$ epidermal growth factor, $5 \mu \mathrm{g} \mathrm{mL}^{-1}$ insulin, $60 \mu \mathrm{g} \mathrm{mL}^{-1}$ bovine pituitary extract (frozen pituitaries from Pel-Freeze, Rogers, AR, U.S.A.) and $0 \cdot 15 \mathrm{mmol} \mathrm{L}^{-1} \mathrm{CaCl}_{2}$. The glucose concentration of $\mathrm{MCDB}$ 153 is $6 \mathrm{mmol} \mathrm{L}^{-1}$ D-glucose. The medium was changed every other day. The growth factors, antibiotics, enzymes and reagents were obtained from Sigma (St Louis, MO, U.S.A.) and the plastic expendables were from Laboratory Science Co. (Corning, NY, U.S.A.).

\section{Fibroblasts}

After removal of the epidermal keratinocytes, fibroblasts were digested from the dermal skin layer using 5\% collagenase for 30 min. Fibroblasts were grown in Dulbecco's modification of Eagle's medium with L-glutamine, sodium pyruvate and pyridoxine (DMEM; Gibco, Grand Island, NY, U.S.A.) supplemented by $10 \%$ fetal calf serum (FCS; Sigma) at $37{ }^{\circ} \mathrm{C}$ in an atmosphere containing $5 \% \mathrm{CO}_{2}$. This medium has a D-glucose concentration of $5.5 \mathrm{mmol} \mathrm{L}^{-1}$. Cells were passaged using trypsin-ethylenediamine tetraacetic acid (Gibco) digestion and were cultured in T-75 $\mathrm{cm}^{2}$ tissue culture flasks.

\section{Fibroblast studies}

Growth of hyperglycaemic fibroblasts in three hyperglycaemic media

After the first passage, fibroblasts were grown in normal ( $\left.5.5 \mathrm{mmol} \mathrm{L}^{-1}\right)$ and three high-glucose (D-glucose)-containing media (hyperglycaemic media), containing 20, 35 and $50 \mathrm{mmol} \mathrm{L}^{-1}$ D-glucose. As a control for the osmotic pressure of the high glucose concentrations, the experiment was repeated using $35 \mathrm{mmol} \mathrm{L}^{-1}$ 'L-glucose' medium (5.5 mmol L ${ }^{-1}$ D-glucose plus $29.5 \mathrm{mmol} \mathrm{L}^{-1}$ L-glucose). All experiments were done using fibroblasts isolated from three different individuals, in triplicate $(\mathrm{n}=3)$. Fibroblasts were cultured in hyperglycaemic medium for 20 days prior to performance of FPCL contraction assays. Fibroblast cultures used in this experiment ranged from two to 14 passages.

\section{Restoration of hyperglycaemic fibroblasts to normal glucose conditions}

To restore hyperglycaemic fibroblasts to normal medium glucose conditions, cell cultures grown in hyperglycaemic media for 15 days were shifted to normal glucose medium for periods of 7 and 21 days.

\section{Preparation of fibroblast-populated collagen lattice}

Type I collagen was extracted from rat tails as described by Bell et al. ${ }^{14}$ FPCL was prepared by a modification of the method of Bell et al. ${ }^{14}$ Briefly, acid-extracted collagen I, fibroblasts, FCS (Sigma), $5 \times$ concentrated DMEM, distilled water and $0.1 \mathrm{~mol} \mathrm{~L}^{-1} \mathrm{NaOH}$ were mixed on ice, so that the final mixture resulted in $0.9 \mathrm{mg} \mathrm{mL}^{-1}$ collagen, $1 \times 10^{5}$ cells $\mathrm{mL}^{-1}$, at physiological ionic strength and $1 \times$ DMEM. Two millilitres of this mixture were poured in 35mm culture dishes (Falcon 1008; Becton Dickinson, Lincoln Park, NJ, U.S.A.). Gelation occurred within $30 \mathrm{~min}$ at $37{ }^{\circ} \mathrm{C}$, at which time $2 \mathrm{~mL}$ of DMEM containing 5\% FCS were overlaid.

\section{Measurement of gel contraction}

Measurement of gel contraction was performed using a modification of the method of Tomasek et al. ${ }^{2}$ Briefly, 3 days of incubation with DMEM containing 5\% FCS were followed by 1 day of serum starvation. The attached lattices were then mechanically released from the underlying substratum by freeing the edges of the collagen lattice with a 27-G syringe needle. Two millilitres of DMEM supplemented by 5\% FCS in different glucose concentrations were immediately added to stimulate contraction. The FPCL contraction, which is one measure of contraction by the fibroblasts embedded in the gel, was quantified by measuring the diameter of two axes of each lattice for up to $3 \mathrm{~h}$ after treatment. As the fibroblasts contract, the diameter of the lattice gel decreases. Each experiment was done in triplicate.

\section{Glutathione treatment of hyperglycaemic fibroblasts}

To determine if esterified GSH (Sigma) corrected the fibroblast contraction defect in hyperglycaemic fibroblasts, cells grown in $35 \mathrm{mmol} \mathrm{L}^{-1}$ glucose were embedded in the collagen lattices for the contraction assay and were treated with $0 \cdot 1,1$, 10 and $100 \mu \mathrm{mol} \mathrm{L}^{-1} \mathrm{GSH}$ for the 4 days prior to the contraction assay. 
Comparison of different antioxidants on fibroblastmediated gel contraction

A single experiment was performed using triplicate cultures from one individual to compare the effect of three antioxidants on fibroblast-mediated collagen gel contraction. GSH ethyl ester $\left(50 \mu \mathrm{mol} \mathrm{L}^{-1}\right)$, N-acetyl-L-cysteine (NAC; $50 \mu \mathrm{mol} \mathrm{L}^{-1}$ ) or pyrrolidinedithiocarbamate (PDC; $50 \mathrm{nmol} \mathrm{L}^{-1}$ to $\left.50 \mu \mathrm{mol} \mathrm{L}{ }^{-1}\right)$ were used to treat high-glucose $\left(35 \mathrm{mmol} \mathrm{L}^{-1}\right)$ fibroblasts. Cultures were treated for 2 days following plating in collagen lattices and some lattices were not treated with antioxidants. Lattices were untreated (control) or treated with 10\% FCS and contraction was stopped by addition of formaldehyde in phosphate-buffered saline (PBS) to a final concentration of $3 \cdot 7 \%$ $1 \mathrm{~h}$ after release. Gel diameters were measured as described.

\section{Morphometric analysis of fibroblast-populated collagen} lattice

Morphometric analysis was performed on cells cultured in hyperglycaemic medium $\left(35 \mathrm{mmol} \mathrm{L}^{-1}\right.$ D-glucose and 'L-glucose') for 20 days. FPCL was prepared with the final cell number being $1 \times 10^{3}$ cells $\mathrm{mL}^{-1}$. Following mechanical release of the collagen gels, measurements were taken before serum stimulation and at 10 and $30 \mathrm{~min}$ after serum stimulation. Collagen lattices were fixed with $4 \%$ formaldehyde and stained with $1 \%$ toluidine blue in PBS containing Tween. After washing three times with PBS, 30 cells were measured in each group by computerized image micrometry. The length of the cells is another measure of the contraction of the fibroblasts in the FPCL assay. Results were evaluated by paired t-test.

\section{Fibroblast viability of fibroblast-populated collagen lattice}

Fibroblast viability at the time of the contraction assay was monitored by using Live/Dead Viability/Cytotoxicity Assay kit, purchased from Molecular Probes (Portland, OR, U.S.A.), according to the manufacturer's protocols. Cultures were examined under the fluorescence microscope by counting three randomly selected fields of view.

\section{Keratinocyte studies}

\section{Growth of primary human keratinocytes in normal and hyperglycaemic media}

Keratinocytes were grown on collagen-coated coverslips in normal $\left(6 \mathrm{mmol} \mathrm{L}^{-1}\right)$, high D-glucose $\left(35 \mathrm{mmol} \mathrm{L}^{-1}\right)$ and high 'L-glucose' (35 $\mathrm{mmol} \mathrm{L}^{-1}$ ) medium for 7 days.

The effect of glutathione treatment $\left(100 \mu \mathrm{mol} \mathrm{L} \mathrm{L}^{-1}\right)$ on apoptotic state of cultured keratinocytes

Apoptosis was used as a measure of a GSH effect on keratinocytes. Apoptosis was quantified using the FragEl (Oncogene,
Cambridge, MA, U.S.A.) (TUNEL assay) kit. Briefly, the cells were fixed with $4 \%$ paraformaldehyde and processed following the kit instructions. The samples were counterstained with methyl green for $10 \mathrm{~min}$. Stained and prepared culture dishes were examined by scanning three randomly selected fields of view under a Nikon TMS inverted phase microscope. Data were expressed as percentage stained cells.

\section{Statistical analysis}

The data are presented as mean \pm SEM. The SEM was used to express variance among samples in studies because cell strains generated from primary cultures developed from a number of individuals (not inbred) were studied. The statistical analysis used unpaired t-test.

\section{Results}

\section{Fibroblasts}

\section{Characteristics of fibroblasts in hyperglycaemic medium}

Growth of fibroblasts in hyperglycaemic medium significantly limited the contraction of the cells in the rapid contraction assay. After addition of the serum, the difference in contraction between fibroblasts in normal medium and in hyperglycaemic medium was statistically significant $(P<0.05)$ at all time points and all media except one, the $180 \mathrm{~min}$, $20 \mathrm{mmol} \mathrm{L}^{-1}$ glucose measurement (Fig. 1a). In contrast, there was no significant difference among the fibroblasts in hyperglycaemic medium in gel contractility at any time point except one: at $180 \mathrm{~min}$, the difference between the $20 \mathrm{mmol} \mathrm{L}^{-1}$ and $35 \mathrm{mmol} \mathrm{L}^{-1}$ glucose hyperglycaemic group was statistically significant $(\mathrm{P}<0.05)$.

Growth of the fibroblasts in 'L-glucose' medium (5.5 mmol L ${ }^{-1}$ D-glucose plus $29.5 \mathrm{mmol} \mathrm{L}^{-1}$ L-glucose) did not affect the ability of the fibroblasts to contract the gels. The contraction was similar to that in the control $5.5 \mathrm{mmol} \mathrm{L}^{-1}$ D-glucose medium plus serum (data not shown).

Irreversibility of the hyperglycaemic effect on fibroblast contraction

The deficiency in contraction of hyperglycaemic fibroblasts was not reversed to normal function by growth of the cells in normal glucose medium for 7 days or 21 days. After 15 days in hyperglycaemic medium, the hyperglycaemic fibroblasts were grown in $5.5 \mathrm{mmol} \mathrm{L}^{-1}$ glucose medium for 7 days (Fig. 1b). The results were similar to those shown in Figure $1 \mathrm{a}$, with significant differences between fibroblasts grown in $5.5 \mathrm{mmol} \mathrm{L}^{-1}$ glucose and in hyperglycaemic media, but with no significant differences within the hyperglycaemic cells $(\mathrm{P}<0.05)$. When the cells were grown in hyperglycaemic medium for 15 days, followed by 21 days at normal glucose levels, the results were similar to those shown in Figure 1a,b, except that in this case (Fig. 1c) the significant differences between normal and 

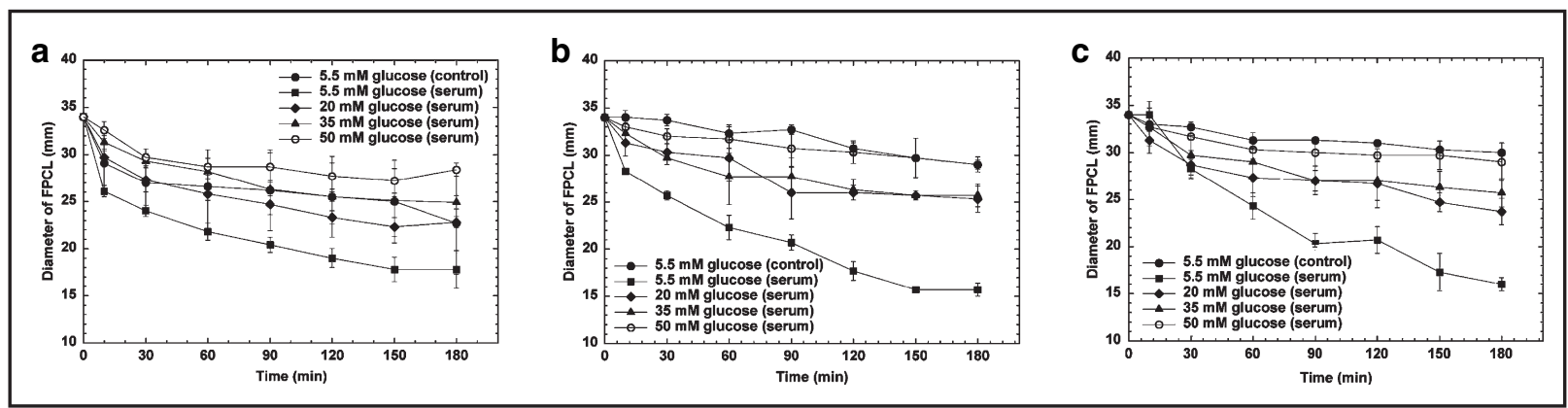

Fig 1. Fibroblast-populated collagen lattice (FPCL) contraction in media containing normal levels of glucose and three different high levels of glucose. (a) The results of FPCL contraction assay. P $<0.05$ at all time points between fibroblasts grown in normal medium and under hyperglycaemic conditions except for cells grown for $180 \mathrm{~min}$ in $20 \mathrm{mmol} \mathrm{L}^{-1}$ glucose. $\mathrm{n}=3$. (b) The results of FPCL contraction assay of fibroblasts which were first grown in normal or high glucose media for 15 days, followed by a 7-day growth period in normal glucosecontaining medium. $\mathrm{P}<0.05$ at all time points between fibroblasts grown in normal medium and under hyperglycaemic conditions. $\mathrm{n}=3$. (c) The results of FPCL contraction assay of cells grown in normal or high glucose media for 15 days, followed by a 21-day growth period in normal glucose-containing medium. $\mathrm{P}<0.05$ between normal and hyperglycaemic cells at the $90-180$-min time points. $\mathrm{n}=3$. Results are presented as mean \pm SEM.

hyperglycaemic medium did not appear until the 90-min time point $(\mathrm{P}<0 \cdot 05)$. For the $90-180$-min time points, the fibroblasts originally grown at a normal glucose level contracted significantly faster than the hyperglycaemic cells, even after 21 days of normal glucose levels in the medium (Fig. 1c).

\section{Glutathione treatment of hyperglycaemic fibroblasts}

GSH treatment restored the ability of hyperglycaemic fibroblasts to contract collagen gels. GSH media concentrations ranging from $0 \cdot 1$ to $100 \mu \mathrm{mol} \mathrm{L}^{-1}$ restored the ability of hyperglycaemic fibroblasts to contract the gels in a concentration-dependent manner. In Figure 2a, collagen lattice contraction profiles are presented for the control cells for the GSH-treated cultures shown in Figure 2b. A comparison of Figure $2 \mathrm{a}$ and Figure $2 \mathrm{~b}$ shows that serum-stimulated $35 \mathrm{mmol} \mathrm{L}^{-1}$ glucose fibroblasts treated with $100 \mu \mathrm{mol} \mathrm{L}{ }^{-1}$ GSH contracted gels to the same degree as serum-stimulated $5.5 \mathrm{mmol} \mathrm{L}^{-1}$ D-glucose cells. Thus $100 \mu \mathrm{mol} \mathrm{L}{ }^{-1} \mathrm{GSH}$ completely overcame the inhibition of contraction induced by fibroblast growth in $35 \mathrm{mmol} \mathrm{L}^{-1}$ hyperglycaemic medium. The data in Figure $2 \mathrm{~b}$ show that this trend was roughly proportional to the GSH concentration in the medium.

\section{Morphometric analysis of serum-stimulated fibroblasts in fibroblast-populated collagen lattice}

\section{Hyperglycaemic cells}

Growth of fibroblasts in hyperglycaemic media did not affect the average length of the cells prior to stimulation. Following serum stimulation the differences among the experimental groups, except for the difference between the 35 and $50 \mathrm{mmol} \mathrm{L}^{-1} \mathrm{D}$-glucose groups, were all statistically significant $(\mathrm{P}<0.05)$ (Fig. 3a).
The hyperglycaemic fibroblasts were grown for 1 week in normal $5.5 \mathrm{mmol} \mathrm{L}^{-1}$ glucose medium (Fig. 3b). Following serum stimulation, the hyperglycaemic fibroblasts failed to contract to the same extent as those grown in $5.5 \mathrm{mmol} \mathrm{L}^{-1}$ glucose throughout. The differences among the experimental groups, except for the difference between the 35 and $50 \mathrm{mmol} \mathrm{L}^{-1} \mathrm{D}$-glucose groups, were all statistically significant $(\mathrm{P}<0.05)$.

\section{Glutathione-treated fibroblasts grown in $35 \mathrm{mmol} \mathrm{L}^{-1}$ glucose}

The average length of the cells grown in $35 \mathrm{mmol} \mathrm{L}^{-1}$ glucose with or without addition of $1 \mu \mathrm{mol} \mathrm{L}{ }^{-1}$ or $100 \mu \mathrm{mol} \mathrm{L} \mathrm{L}^{-1}$ GSH (Fig. 3c) was similar before serum stimulation. At $30 \mathrm{~min}$ after stimulation, the difference between the untreated and $1 \mu \mathrm{mol} \mathrm{L}{ }^{-1}$ GSH-treated groups was statistically insignificant $(\mathrm{P}>0 \cdot 1)$, whereas at both 10 and $30 \mathrm{~min}$ the differences between cultures treated with $100 \mu \mathrm{mol} \mathrm{L}^{-1} \mathrm{GSH}$ and untreated hyperglycaemic cells were significant $(\mathrm{P}<0.05)$.

\section{Effect of other antioxidants on fibroblast lattice contraction}

The data in Figure 4 show that both GSH ethyl ester and NAC treatment resulted in significantly greater $(P<0.05)$ contraction when compared with the control (no antioxidant, $35 \mathrm{mmol} \mathrm{L}^{-1}$ glucose plus $10 \%$ serum), and that GSH treatment caused significantly more contraction than did NAC treatment $(\mathrm{P}<0.05)$. PDC caused no significant enhancement of contraction at any concentration used. Higher concentrations of PDC resulted in reduced contraction. Under microscopic examination of these cultures, the cells appeared rounded and did not develop the typical spindle-shaped morphology of fibroblasts cultured in collagen. 


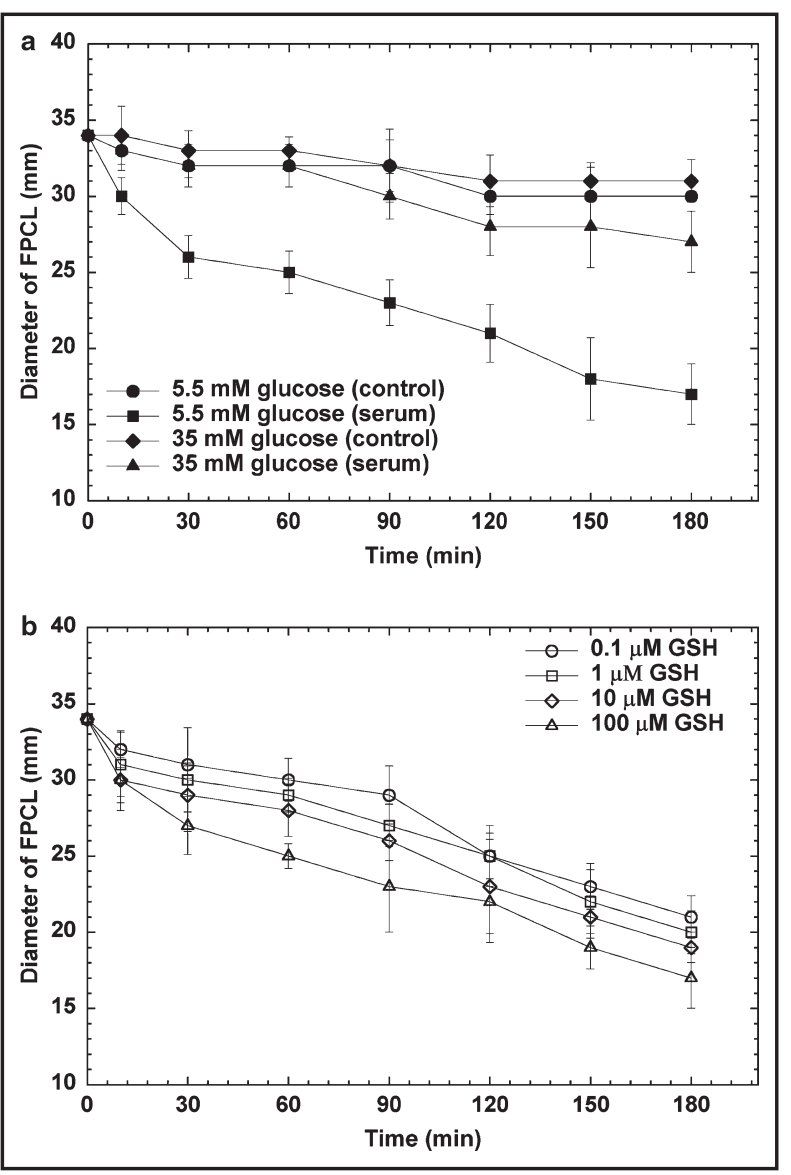

Fig 2. The effect of glutathione (GSH) on fibroblast-populated collagen lattice (FPCL) contraction of hyperglycaemic fibroblasts. (a) Control lattice. $\mathrm{P}<0.05$ between $5.5 \mathrm{mmol} \mathrm{L}^{-1}$ glucose plus serum and $5.5 \mathrm{mmol} \mathrm{L}^{-1}$ glucose with no serum, and $35 \mathrm{mmol} \mathrm{L}^{-1}$ glucose with and without serum. $n=3$. (b) The results of FPCL contraction assay when fibroblasts grown in $35 \mathrm{mmol} \mathrm{L}^{-1}$ glucose plus serum were treated with $0 \cdot 1-100 \mu \mathrm{mol} \mathrm{L}^{-1}$ esterified GSH after 7 days. $\mathrm{P}<0.05$ significance for all $100 \mu \mathrm{mol} \mathrm{L} \mathrm{L}^{-1} \mathrm{GSH}$ time points, and for all concentrations of GSH at the 120-, 150- and 180-min time points, when compared with their respective controls in panel (a). $\mathrm{n}=3$. Results are presented as mean \pm SEM.

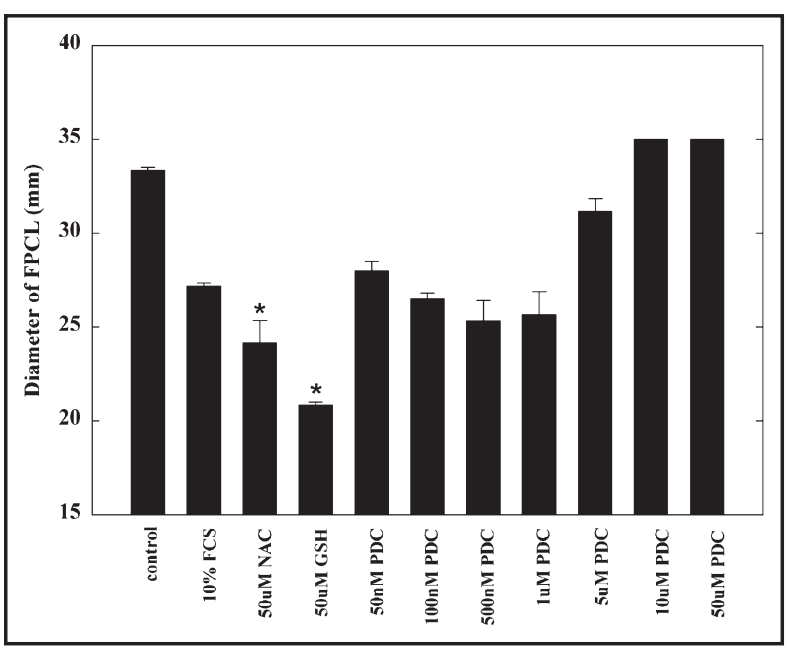

Fig 4. Effect of other antioxidants on fibroblast-populated collagen lattice (FPCL) contraction. Both glutathione (GSH) and N-acetyl-Lcysteine (NAC) treatment resulted in significantly greater $(* \mathrm{P}<0.05)$ contraction when compared with the control (no antioxidant, $35 \mathrm{mmol} \mathrm{L}^{-1}$ glucose plus $10 \%$ serum). Pyrrolidinedithiocarbamate (PDC) caused no significant enhancement of contraction at any concentration used, and higher concentrations of PDC resulted in reduced contraction. Results are presented as mean $\pm \mathrm{SD}$.

Viability of fibroblasts after fibroblast-populated collagen lattice assay

The difference in the percentage of live cells among the fibroblasts grown in hyperglycaemic conditions and in $5.5 \mathrm{mmol} \mathrm{L}^{-1}$ glucose was statistically insignificant before stimulation and at both 10 and 30 min after serum stimulation. The percentage of live cells was over $95 \%$ in all groups at every time point (data not shown).

\section{Apoptosis in hyperglycaemic fibroblasts}

Determination of the percentage of apoptotic fibroblasts was performed at three different time points following addition of
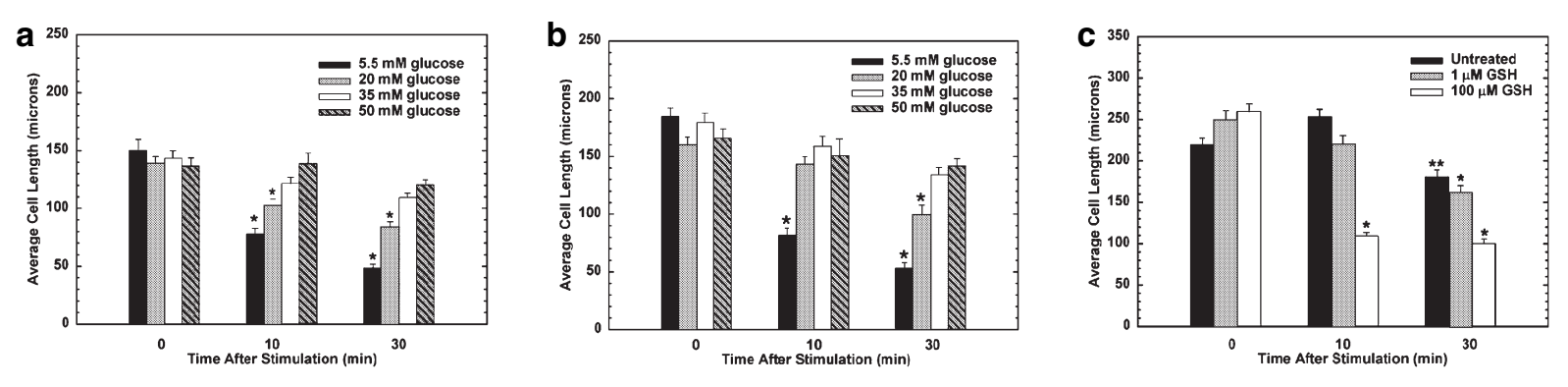

Fig 3. Morphometric analysis of fibroblast-populated collagen lattice fibroblasts. (a) The average cell length of hyperglycaemic cells (35 mmol L ${ }^{-1}$ glucose) before stimulation, and at 10 and 30 min after stimulation with $5 \%$ serum. $* \mathrm{p}<0.05, \mathrm{n}=3$. (b) The average cell length of hyperglycaemic cells $\left(35 \mathrm{mmol} \mathrm{L}^{-1}\right.$ glucose) following 1 week of treatment with normal glucose-containing medium before stimulation, and at 10 and 30 min after stimulation with $5 \%$ serum. $* \mathrm{P}<0 \cdot 05, \mathrm{n}=3$. (c) The average cell length of hyperglycaemic cells $\left(35 \mathrm{mmol} \mathrm{L}^{-1}\right.$ glucose) treated with 1 and $100 \mu \mathrm{mol} \mathrm{L}{ }^{-1}$ esterified glutathione (GSH) before stimulation, and at 10 and 30 min after stimulation with $5 \%$ serum. $* \mathrm{P}<0.05, * * \mathrm{P}<0.01, \mathrm{n}=3$. Results are presented as mean $\pm \mathrm{SD}$. 


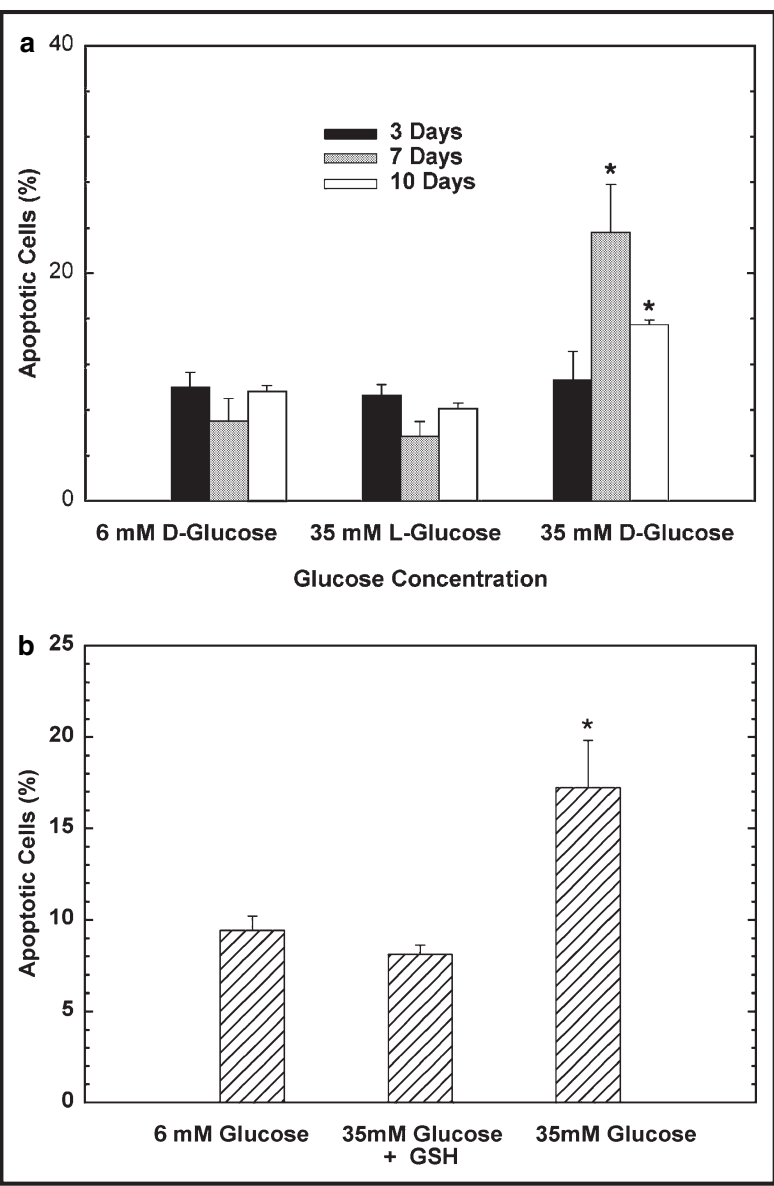

Fig 5. Keratinocyte apoptosis. (a) The percentage of apoptotic keratinocytes following exposure to normal $\left(6 \mathrm{mmol} \mathrm{L}^{-1}\right)$ D-glucose and high $\left(35 \mathrm{mmol} \mathrm{L}^{-1}\right) \mathrm{D}$ - and L-glucose at 3, 7 and 10 days. $* \mathrm{P}<0 \cdot 05$. (b) The percentage of apoptotic keratinocytes following esterified glutathione (GSH) treatment in medium containing high D-glucose at 7 days. $* \mathrm{P}<0.05$. Results are presented as mean $\pm \mathrm{SD}$.

high levels of glucose. The percentage of apoptotic cells in media containing normal $\left(5.5 \mathrm{mmol} \mathrm{L}^{-1}\right)$ D-glucose, $35 \mathrm{mmol} \mathrm{L}^{-1}$ 'L-glucose' and $35 \mathrm{mmol} \mathrm{L}^{-1}$ D-glucose showed no differences at 3, 7 and 10 days (data not shown). Thus neither L- nor D-glucose induced apoptosis in adult human fibroblasts.

\section{Keratinocytes}

\section{Apoptosis in hyperglycaemic keratinocytes}

Comparisons of the percentage of apoptotic cells were made after 3, 7 and 10 days (Fig. 5a). The difference in the percentage of apoptotic cells following 3 days of growth in media containing normal $\left(6 \mathrm{mmol} \mathrm{L}^{-1}\right)$ D-glucose, $35 \mathrm{mmol} \mathrm{L}^{-1}$ 'L-glucose' and $35 \mathrm{mmol} \mathrm{L}{ }^{-1}$ D-glucose was not significant $(P>0 \cdot 1)$. After 7 and 10 days, the number of apoptotic cells was increased in the keratinocytes grown in $35 \mathrm{mmol} \mathrm{L}^{-1}$ D-glucose $(\mathrm{P}<0.05)$ but was unaltered in those grown in $35 \mathrm{mmol} \mathrm{L}^{-1}$ 'L-glucose'. This supports the premise that the effect of glucose is not due to osmotic changes caused by the addition of glucose to the medium.

Apoptosis in glutathione-treated keratinocytes grown in $35 \mathrm{mmol} \mathrm{L}^{-1}$ glucose medium

The effect of esterified GSH treatment was determined at 7 days because the observed difference in apoptosis was greatest at this time. Esterified GSH was added with the hyperglycaemic medium and continued for the entire 7 days. The control groups were grown in normal and $35 \mathrm{mmol} \mathrm{L}^{-1}$ 'L-glucose' medium. The difference between GSH-treated and untreated hyperglycaemic cells was statistically significant $(\mathrm{P}<0.05)$. However, the difference between the normal and GSH-treated $35 \mathrm{mmol} \mathrm{L}^{-1}$ glucose groups was not $(\mathrm{P}>0 \cdot 1)$ (Fig. 5b).

\section{Discussion}

Impaired healing of acute wounds and increased susceptibility to chronic nonhealing wounds are an undeniable reality for patients with diabetes. Successful treatment modalities have focused on maintaining a clean, moist wound bed through debridement and antibiotic treatment. More recently, the application of exogenous growth factors including acidic fibroblast growth factor, keratinocyte growth factor, transforming growth factor- $\beta_{1}$, platelet-derived growth factor (PDGF), PDGF-BB and various agents including antioxidants has been reported to improve wound healing in both normal and delayed wound healing conditions. ${ }^{1,16-22}$ Although studies have shown the importance of several growth factor treatment modalities, the use of topical delivery systems is still controversial. These studies suggest that cells under oxidative stress do not function well, and that the response to mitogenic and stimulatory factors occurs most efficiently when cells are in oxidative stress-free conditions.

The hypothesis was that delayed wound healing in the diabetic patient results from the presence of a functional defect in both skin fibroblast populations and in keratinocyte populations, so that the cells do not respond appropriately to normal stimuli. These defects would result in impaired wound contraction and reduced epithelialization. Loss of cellular communication among skin cells in the healing wound due to a reduced number of keratinocytes may also be detrimental to successful wound healing.

Thus, the first tissue culture models to examine the effects of hyperglycaemic medium on physiologically relevant functions of the two predominant skin cell types involved in dermal healing were developed by us. Because the predominant force behind wound closure is fibroblast-mediated contraction, the effect of hyperglycaemic medium on FPCL contraction was examined. Fibroblasts cultured for a prolonged period in hyperglycaemic medium demonstrated a lessened ability to contract collagen gels. The ability to contract collagen was inversely related to the concentration of glucose in the culture medium in which the cells were grown. Furthermore, the change was not 
reversed by resumption of culture in normal glycaemic medium, at least over the 3 -week time period that was studied.

Our data suggest that the lack of contraction in these cells was not due to progressive accumulation of glycation end products. A portion of the medium L-glucose would enter the cells using passive transport while the bulk would remain in the medium, as passive transport is a slow process. As glycation is nonenzymatic, both D- and L-glucose should act as substrate, whether in the medium or in the cell cytoplasm. Hyperglycaemic medium using L-glucose to augment the medium glucose concentration did not affect fibroblast cell contraction or collagen gel contraction, while hyperglycaemic medium consisting entirely of D-glucose affected each parameter of fibroblast function that was studied. These data suggest that there is a phenotypic change in fibroblasts cultured in hyperglycaemic medium that results in a defect in cellular contraction. This change could result in a loss of ability to process signals initiating contraction or in a loss of ability to contract in response to those signals.

The generation of tension in granulation tissue and subsequently by fibroblasts undergoing contraction is believed to be a cell-mediated event. ${ }^{2,13}$ Rapid contraction of FPCL is suggested to be dependent on the shortening of the elongated fibroblasts and compaction of the stress fibres. Using cellular morphometric analysis, it was found that that the shortening of fibroblasts in differential degrees of glucose significantly affects the ability of fibroblasts to contract in the collagen gels. Several authors have shown that the contraction of FPCL is directly proportional to the number of cells in the gel construct. $^{2,23}$ It has also been suggested that the reorganization of collagen fibrils by resident fibroblasts contributes to the FPCL contraction. $^{24}$ The defect in collagen contraction, as reported here, may partially result from impaired fibroblast reorganization of collagen in addition to the defect in fibroblast contraction, as there was no observed change in cell number.

Experimental and clinical studies have reported that high glucose levels caused significant increases in apoptosis in endothelial cells, while fibroblasts appeared more resistant to apoptosis. $^{8,9}$ Our data support this conclusion.

The effect of hyperglycaemia on cultured keratinocytes was then investigated. Our studies with keratinocytes showed that this cell type readily undergoes enhanced apoptosis when grown in hyperglycaemic medium for a period longer than 3 days. It is likely that cells undergoing apoptosis cannot modulate the interactions required for normal wound healing. Even though the percentage of apoptotic keratinocytes was higher in hyperglycaemic medium, it is also possible that the hyperglycaemic keratinocyte will not produce the molecular signals necessary for normal wound healing.

Cutaneous wound healing is delayed in patients with diabetes, probably because of increased oxidative stress ${ }^{1,5,6}$ and variations in cellular GSH levels. The balance between GSH and GSSG is essential to maintain cellular homeostasis. The protective effects of GSH can occur by direct scavenging of reactive oxygen species, scavenging of lipid peroxidation products catalysed by GSH peroxidases, preserving thiol-disulphide status of proteins and acting as an essential component in repair of oxidant damage. ${ }^{14,25-29}$

Our data showed that fibroblasts grown in hyperglycaemic medium have a significant contraction deficiency in a doseand time-dependent manner. Treatment with esterified GSH was able to reverse the defect in contraction function, as did NAC, although another oxidant (PDC) was ineffective, possibly due to cell toxicity. Hyperglycaemia-induced keratinocyte apoptosis was also rescued by GSH treatment.

In summary, the contraction defect of fibroblasts in an in vitro model appears to mimic diabetes as a cellular event. The cause of the defect seems, at least in part, to result from depressed intracellular GSH. It is likely that lack of wound contraction in patients with diabetes is related to the observed defect in fibroblast contraction.

Our data also demonstrated that keratinocytes in hyperglycaemic medium are more susceptible to apoptosis and that treatment with esterified GSH rescues the keratinocytes from apoptosis. Our data suggested that the cultured cell models used in our study are relevant to the in vivo disease state and that this defect can be corrected by supplementation of esterified GSH, at least in vitro. GSH is known to play a major role in the regulation of cellular integrity, proliferation and protein function, and therefore disruption of GSH homeostasis may be a key event leading to delayed wound healing. It is possible that treatment modalities to restore intracellular GSH level in patients with diabetes could aid the healing of diabetic wounds.

\section{Acknowledgments}

This work was supported by grant NIAMS AR 26009.

\section{References}

1 Mellin TN, Cashen DE, Ronan JJ et al. Acidic fibroblast growth factor accelerates dermal wound healing in diabetic mice. J Invest Dermatol 1995; 103:850-5.

2 Tomasek JJ, Haaksma CJ, Eddy RJ, Vaughan MB. Fibroblast contraction occurs on release of tension in attached collagen lattices: dependency on an organized actin cytoskeleton and serum. Anat Rec 1992; 232:359-68.

3 Germain L, Jean A, Auger FA, Garrel DR. Human wound healing fibroblasts have greater contractile properties than dermal fibroblasts. J Surg Res 1994; 57:268-73.

4 Obara K, Nikcevic G, Pestic L et al. Fibroblast contractility without an increase in basal myosin light chain phosphorylation in wild type cells and cells expressing the catalytic domain of myosin light chain kinase. J Biol Chem 1995; 270:18734-7.

5 Hehenberger K, Heilborn JD, Brismar K, Hansson A. Inhibited proliferation of fibroblasts derived from chronic diabetic wounds and normal dermal fibroblasts treated with high glucose associated with increased formation of L-lactate. Wound Repair Regen 1998; 6:135-41.

6 Morocutti A, Sethi M, Hayward A et al. Glutathione reverses the growth abnormalities of skin fibroblasts from insulin-dependent diabetic patients with nephropathy. J Am Soc Nephrol 1998; 9:1060-6.

7 Cheng H, Feldman EL. Bidirectional regulation of p38 kinase and c-jun N-terminal protein kinase by insulin-like growth factor-I. J Biol Chem 1998; 273:14560-5. 
8 Baumgartner-Parzer SM, Wagner L, Petterman M et al. High-glucose-triggered apoptosis in cultured endothelial cells. Diabetes 1995; 44:1323-7

9 Mehlen P, Schulze-Osthoff K, Arrigo A. Small stress proteins as novel regulators of apoptosis. J Biol Chem 1996; 271:16510-14.

10 Gavrieli Y, Sherman Y, Ben-Sasson SA. Identification of programmed cell death in situ via specific labeling of nuclear DNA fragmentation. J Cell Biol 1992; 119:493-501.

11 Kashiwagi A, Asahina T, Nishiho Y et al. Glycation, oxidative stress, and scavenging activity. Diabetes 1996; 45 (Suppl. 3):S84-6.

12 Shan XQ, Aw TY, Jones DP. Glutathione-dependent protection against oxidative injury. Pharmacol Ther 1990; 47:61-71.

13 Mudge B, Harris C, Rees R. Altered glutathione status in two examples of chronic human wounds. Surg Forum 1998; 49:629.

14 Bell E, Ivarsson B, Merrill C. Production of a tissue like structure by contraction of collagen lattices by human fibroblasts of different differential potential in vitro. Proc Natl Acad Sci USA 1979; 76:1274-8.

15 Marcelo CL, Duel EA, Rhodes LM, Dunham WR. An in vitro model of essential fatty acid deficiency. J Invest Dermatol 1992; 99:771-7.

16 Kuzuya M, Satake S, Ai S et al. Inhibition of angiogenesis on glycated collagen lattices. Diabetologia 1998; 41:491-9.

17 Puolakkainen PA, Twardzik DR, Ranchalis JE et al. The enhancement in wound healing by transforming growth factor- $\beta 1$ (TGF- $\beta 1$ ) depends on the topical delivery system. Ann Surg Res 1995; 58:3219.

18 Heggers JP, Elzaim H, Garfield R et al. Effect of the combination of Aloe vera, nitroglycerin, and L-NAME on wound healing in the rat excisional model. J Altern Complement Med 1997; 3:149-53.

19 Shukla A, Rasik AM, Patnaik GK. Depletion of reduced glutathione, ascorbic acid, vitamin $\mathrm{E}$ and antioxidant defence enzymes in a healing cutaneous wound. Free Radic Res 1997; 26:93-101.

20 Pierce GF, Vande Berg J, Rudolph R et al. Platelet-derived growth factor-bb and transforming growth factor beta 1 selectively modu- late glycosaminoglycans, collagen, and myofibroblasts in excisional wounds. Am J Pathol 1991; 138:629-46.

21 Robson MC, Phillips LG, Thomason LE et al. Platelet-derived growth factor-BB for the treatment of chronic pressure ulcers. Lancet 1992; 339:23-5.

22 Liechty KW, Nespit M, Herlyn M et al. Adenoviral-mediated overexpression of platelet-derived growth factor-b corrects ischemic impaired wound healing. J Invest Dermatol 1999; 113:375-83.

23 Erlich HP, Cremona O, Gabbiani G. The expression of $\alpha 2 \beta 1$ integrin and $\alpha$-smooth muscle actin in fibroblasts grown on collagen. Cell Biochem Func 1998; 16:129-37.

24 Asaga H, Kukichi S, Yoshizato K. Collagen gel contraction by fibroblasts requires cellular fibronectin but not plasma fibronectin. Exp Cell Res 1991; 193:167-74.

25 Malinda KM, Sidhu GS, Mani $\mathrm{H}$ et al. Thymosin $\beta 4$ accelerates wound healing. J Invest Dermatol 1999; 113:364-8.

26 Asensi M, Sastre J, Pallardo FV et al. Ratio of reduced to oxidized glutathione as indicator of oxidative stress status and DNA damage. Methods Enzymol 1997; 299:267-76.

27 Urata Y, Yamamoto H, Goto S et al. Long exposure to high glucose concentration impairs the responsive expression of $\gamma$-glutamylcystein synthetase by interleukin- $1 \beta$ and tumor necrosis factor $\alpha$ in mouse endothelial cells. J Biol Chem 1999; 271:15146-52.

28 Finco TS, Beg AA, Baldwin AS. Inducible phosphorylation of I kappa B alpha is not sufficient for its dissociation from NF- $\kappa \mathrm{B}$ and is inhibited by protease inhibitors. Proc Natl Acad Sci USA 1994; 91:11884-8.

29 Nakayama K, Shimizu H, Mitomo K et al. A lymphoid cell-specific nuclear factor containing c-rel-like proteins preferentially interacts with interleukin-6 kappa b-related motifs whose activities are repressed in lymphoid cells. Mol Cell Biol 1999; 12:1736-46. 